\title{
Mitochondrial effects of Ginkgo biloba extract
}

\author{
Anne Eckert \\ Neurobiology Laboratory for Brain Aging and Mental Health, Psychiatric University Clinics, Basel, Switzerland
}

ABSTRACT

Oxidative stress and mitochondrial failure promote altered protein degradation, reduced neurotransmission, synapse loss and tau/hyperphosphorylation, which are early stages in the development of Alzheimer's disease (AD). A growing volume of data confirms that Ginkgo biloba extract (GBE) reduces oxidative stress and improves mitochondrial respiration and thus may be useful in preventing or slowing down the progression of AD. Treatment of Caenorhabditis elegans with GBE- extract reduces oxidative stress and extends median lifespan compared with controls. Levels of reactive oxygen species, including the superoxide anion radical, were reduced in brains from GBE-treated mice compared with controls. In older mice, GBE resulted in a protective effect by increasing production of adenosine triphosphate in neurons. A respiratory experiment indicated that GBE was able to rescue $\mathrm{A} \beta$-induced defects in energy metabolism, with results suggesting longterm regulatory effects on mitochondria. GBE also had a selective effect on the activities of mitochondrial enzymes that assemble the electron transport system. The flavonoids, bilobalide and some of the ginkgolides (B and J) had a high protective capacity, indicating that a combination of several compounds within standardized Gingko biloba extracts contribute disproportionately for these protective effects.

Key words: adenosine triphosphate (ATP), Alzheimer's disease, antioxidant, Ginkgo biloba, membrane potential, mitochondria, mitochondrial dysfunction, oxidative stress, reactive oxygen species

\section{Introduction}

Much of the current research into treatments for Alzheimer's disease (AD) focuses on dysfunction of the amyloid cascade, which is traditionally considered to be a linear sequence of events (Figure 1). Certain events are active earlier in the disease course: for example, oxidative stress and mitochondrial failure promote altered protein degradation, reduced neurotransmission, accelerate synapse loss and tau-hyperphosphorylation. Triggers such as genetics, age and environment may affect processing of amyloid precursor protein (APP) and hence generation of beta amyloid $(\mathrm{A} \beta)$, which aggregates into amyloid oligomers and fibrils and finally their deposition as plaques. Each event in the amyloid cascade is an important trigger for the changes that ultimately result in $\mathrm{AD}$. Some agents may be able to interfere with this cascade, providing a degree of neuroprotection from the development of this condition.

Correspondence should be addressed to: Anne Eckert, Neurobiology Laboratory for Brain Aging and Mental Health, Assoc. Res. Group Dept. Biomedicine Univ. of Basel, Psychiatric University Clinics Basel, Wilhelm Klein-Strasse 27, CH-4025 Basel, Switzerland. Phone: +41 6132554 87, Fax: +41 6132555 77. Email: anne.eckert@upkbs.ch.

\section{Oxidative stress and mitochondrial failure}

Wu and colleagues used the model organism Caenorhabditis elegans to evaluate the pharmacological effects of standardized Gingko biloba leaf special extract EGb $761^{\circledR}$ on aging and to test the hypothesis that this extract augments the natural antioxidant system of $C$. elegans (Wu et al., 2002). The results showed that treatment of wild-type worms with EGb 761 reduces oxidative stress and also extends median lifespan compared with controls. EGb 761 is a dry extract from Ginkgo biloba leaves (35-67:1), extraction solvent acetone $60 \%(\mathrm{w} / \mathrm{w})$. The extract is adjusted to $22.0-27.0 \%$ ginkgo flavonoids and $5.0-7.0 \%$ terpene lactones, consisting of $2.8-3.4 \%$ ginkgolides $\mathrm{A}, \mathrm{B}$, and $\mathrm{C}$ and $2.6-3.2 \%$ bilobalide and contains less than $5 \mathrm{ppm}$ ginkgolic acids.

Mitochondria are producers of reactive oxygen species (ROS), and dysfunction of the mitochondria has a central role in aging and is an early event in neurodegenerative diseases (Abdel-Kader et al., 2007). Aged mice (18 months old) were treated with EGb 761 for 14 days and the cortical brain cells were then investigated post-mortem for the presence of ROS using fluorescent dyes. Levels of ROS and particularly the superoxide anion radical $\mathrm{O}_{2}{ }^{-}$(an aggressive form of ROS formed as an 


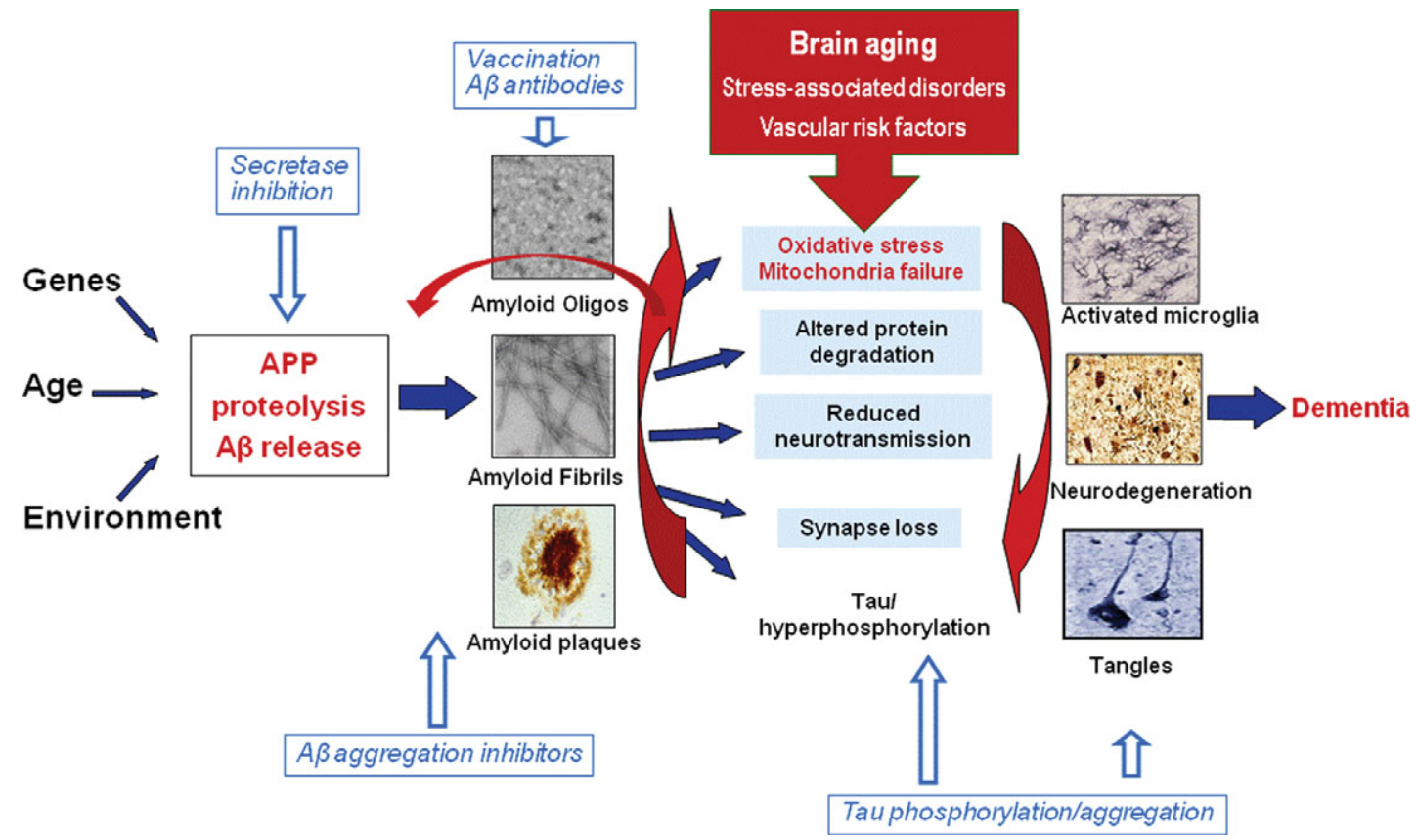

Figure 1. Amyloid- $\beta$ cascade hypothesis and potential intervention strategies.

unwanted by-product of mitochondrial respiration) were reduced in brain cells from EGb 761treated mice compared with controls (placebotreated mice). EGb 761 thus provides antioxidant effects.

A high mitochondrial membrane potential is vital for the production of adenosine triphosphate (ATP), which has an important role in intracellular energy transfer. In a study by Eckert et al. (2005) in aged mice (22 months old), brain cells were isolated and subjected to oxidative stress through the application of hydrogen peroxide. Control mice exhibited a consequent reduction in membrane potential, which was followed by a reduction in production of ATP. In comparison, brain cells from mice treated with Ginkgo biloba extract EGb 761 were protected from the effects of hydrogen peroxide, which had a reduced effect on ATP production. Shi et al. (2010) also investigated the impact of EGb 761 on ATP production using SAMP8 mice (a senescence-accelerated strain of mouse). The hippocampi of two age groups of mice ( 3 weeks and 40 weeks) were isolated and the ATP content in the mitochondria was determined. In young mice, EGb 761 had no effect on basal production of ATP; however, treatment of older mice with $\mathrm{EGb} 761$ resulted in a protective effect, with levels of ATP increasing.

A respiratory experiment in a closed chamber investigated whether standardized Ginkgo biloba extract was able to rescue $\mathrm{A} \beta$-induced defects in energy metabolism using whole cells (Rhein et al., 2010). In this experiment, the respiratory capacity of human neuroblastoma cells overexpressing human wild-type amyloid precursor protein (APP) and control cells (stably transfected with the empty vector) was examined under physiological conditions before and after treatment with standardized Ginkgo biloba extract for 24 hours. The oxygen concentration in the chamber decreases once mitochondria begin to consume oxygen and oxygen consumption (also called mitochondrial respiration) can be measured. When compared to control cells, APP transfected cells which chronically overproduce $\mathrm{A} \beta$ showed a strong reduction in respiratory capacity and coupling of the mitochondrial electron transport chain. For cells treated with Gingko biloba extract, however, a general antioxidant effect led to an increase in energy homeostasis and a reduction in ROS levels in both control and APP cells. Importantly, respiration remained improved in the Ginkgo biloba extract-treated cells even after removal of the extract, which suggests that the extract has longterm regulatory effects on mitochondria. The activities of mitochondrial enzymes that assemble the electron transport system (ETS) were also examined, with interesting results. Complex I activity was significantly increased after APP cells but not control cells were treated with Ginkgo biloba extract (Figure 2A), whereas Complex II activity was unaffected by Ginkgo biloba extract in both cell lines. Complex III activity was markedly increased in APP cells but normalized to the level of control cells after treatment with Ginkgo biloba extract (Figure 2B). Complex IV activity was decreased 

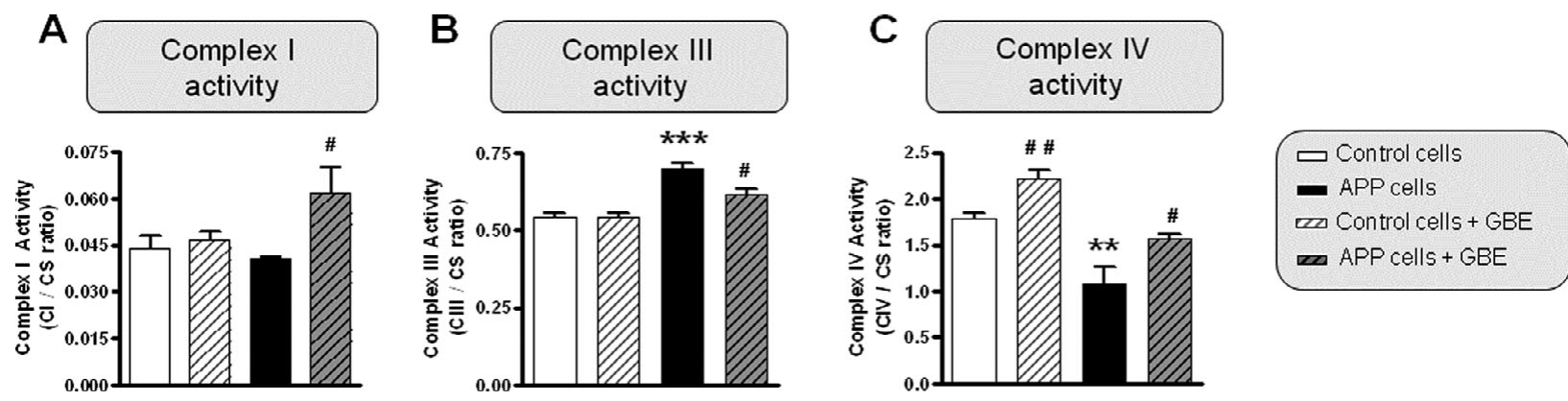

Figure 2. Modulation of mitochondrial enzyme activities by Gingko biloba extract in control and amyloid precursor protein (APP) cells. Reproduced with permission from Rhein et al., 2010.

Table 1. Protective capacity of individual compounds of EGb 761 in brain cells $(n=6-12)$

\begin{tabular}{lc}
\hline $\begin{array}{l}\text { COMPOUND } \\
(0.5 \mathrm{mg} / \mathrm{ml})\end{array}$ & $\begin{array}{l}\text { MEAN (SEM) PROTECTION (\%) } \\
\text { (percentage recovery after insult) }\end{array}$ \\
\cline { 1 - 2 } Ginkgolide A & $8.0 \pm 1.9^{* *}$ \\
Ginkgolide B & $33.0 \pm 11.2^{*}$ \\
Ginkgolide C & $8.5 \pm 1.6^{* *}$ \\
Ginkgolide J & $37.0 \pm 12.2^{*}$ \\
Bilobalide & $36.3 \pm 7.7^{* *}$ \\
Flavonoids & $56.4 \pm 12.7^{* * *}$ \\
\hline
\end{tabular}

$\mathrm{SEM}=$ standard error mean.

${ }^{*} \mathrm{P}<0.05$ versus no insult; ${ }^{* *} \mathrm{P}<0.01$ versus no insult; ${ }^{* * *} \mathrm{P}<0.001$ versus no insult.

in APP cells but significantly increased in control and APP cells after treatment with Ginkgo biloba extract (Figure 2C). The reason for the specific effect of the drug on activity of the different mitochondrial enzymes is unclear, but it may relate to normalization of the $\mathrm{A} \beta$-induced disturbance in the activity of complexes III and IV.

When the effects of the component compounds of Ginkgo biloba extract on membrane protection were compared, the flavonoids, bilobalide and some of the ginkgolides ( $\mathrm{B}$ and $\mathrm{J}$ ) had a high protective capacity (Table 1 ), which indicates that a combination of several compounds within the extract may contribute disproportionately for its protective effects. This is interesting, because the extract has a large effect on the mitochondrial DNA compared to levels in controls and APPtransected cells. This may indicate that activity of certain complexes is increased and is important for protective effects in terms of mitochondrial dysfunction.

\section{Summary}

A meaningful selection of data confirms that Ginkgo biloba extract has beneficial effects in terms of reducing oxidative stress and improving mitochondrial respiration, which may be useful in preventing the early stages of $\mathrm{AD}$.

\section{Conflict of interest}

The author has undertaken consultancy services for Dr Willmar Schwabe GmbH \& Co KG, Karlsruhe, Germany.

\section{References}

Abdel-Kader, R. et al. (2007). Stabilization of mitochondrial function by Ginkgo biloba extract (EGb 761). Pharmacological Research, 56, 493-502.

Eckert, A., Keil, U., Scherping, I., Hauptmann, S. and Müller, W. E. (2005). Stabilization of mitochondrial membrane potential and improvement of neuronal energy metabolism by Ginkgo biloba extract EGb 761. Annals of the New York Academy of Sciences, 1056, 474-85.

Rhein, V. et al. (2010). Ginkgo biloba extract ameliorates oxidative phosphorylation performance and rescues abeta-induced failure. PLoS One, 5 (8), e12359.

Shi, C. et al. (2010). Ginkgo biloba extract EGb761 protects against aging-associated mitochondrial dysfunction in platelets and hippocampi of SAMP8 mice. Platelets, 21, 373-379.

Wu, Z. et al. (2002). Ginkgo biloba extract EGb 761 increases stress resistance and extends life span of Caenorhabditis elegans. Cellular and Molecular Biology (Noisy-le-Grand, France), 48, 725-731. 